\title{
Extrapancreatic solid pseudopapillary tumors: A clinicopathological analysis of two cases
}

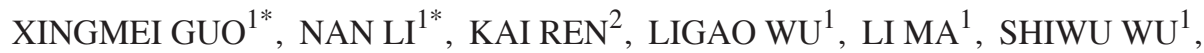 \\ FENGMEI XIE $^{1}$ and ZHENZHONG FENG ${ }^{1}$
}

\author{
${ }^{1}$ Department of Pathology, The First Affiliated Hospital of Bengbu Medical College, Bengbu Medical College, \\ Bengbu, Anhui 233000; ${ }^{2}$ Faculty of Pharmacy, Bengbu Medical College, Bengbu, Anhui 233004, P.R. China
}

Received August 7, 2015; Accepted February 9, 2016

DOI: $10.3892 /$ mco.2016.802

\begin{abstract}
Solid pseudopapillary tumors (SPTs) are unusual neoplasms that mostly occur in the pancreas, and predominantly affect young women. As a low-grade malignant neoplasm of the exocrine pancreas, they occasionally metastasize, usually to the liver or peritoneum. It has been reported that $<1 \%$ of SPTs are primary extrapancreatic SPTs. In the present study, we present two rare, but conspicuous extrapancreatic SPTs. Both occurred in young women, and showed good prognoses following surgery. One was a recurrent SPT of the pancreas that metastasized to the ovary, and the other was a distinct primary neoplasm that arose in the retroperitoneal area. The pathological features of the two tumors, including solid and pseudopapillary growth patterns with pale or eosinophilic cytoplasm, were characteristic of SPTs of the pancreas. However, in the case of the metastatic ovarian tumor, focal necrosis and an increased nuclear-to-cytoplasmic ratio were observed. The presence of positive nuclear-cytoplasmic $\beta$-catenin, the loss of membranous E-cadherin expression, and a perinuclear punctate CD99 staining pattern on immunohistochemistical analysis, were essential features for diagnosis. The aim of the present study was to compare the morphological and immunohistochemical features of these tumors with those typical of pancreatic SPTs, and to raise awareness that SPTs are able to metastasize to
\end{abstract}

Correspondence to: Professor Zhenzhong Feng, Department of Pathology, The First Affiliated Hospital of Bengbu Medical College, Bengbu Medical College, 287 Changhuai Road, Bengbu, Anhui 233000, P.R. China

E-mail: 13455367621@163.com

*Contributed equally

Abbreviations: SPT, solid pseudopapillary tumor; CA, carbohydrate antigen; N/C, nuclear-to-cytoplasm; YST, yolk sac tumor; EMA, epithelial membrane antigen; AACT, $\alpha 1$-antichymotrypsin; Syn, synaptophysin; CgA, chromogranin A; ER, estrogen receptor; PR, progesterone receptor

Key words: extrapancreatic, metastasis, ovarian, retroperitoneal, solid pseudopapillary tumor unusual sites, and may also arise as primary tumors outside the pancreas, which may lead to diagnostic dilemmas.

\section{Introduction}

Solid pseudopapillary tumors (SPTs) are rare, indolent tumors that mostly occur in the pancreas, and account for $0.1-3 \%$ of all exocrine pancreatic tumors (1). This type of neoplasm was first named after V. K. Frantz, who identified its characteristics in 1959 (2). It was subsequently reported under various names, until the term 'solid pseudopapillary tumor (or neoplasm)' was finally adopted by the World Health Organization (WHO) in 1996 (3). SPTs are defined as low-grade malignant neoplasms of the exocrine pancreas in the current WHO classification (4). There have been 2,744 patients with SPTs identified in 484 studies published between 1961 and 2012, according to a systematic review of SPTs by Law et al (5). The number of SPTs reported in the literature has increased 7-fold since 2000 compared with the previous decades (5). The disease typically occurs in young women, and possesses unique clinicopathological features. Most patients initially present with abdominal pain or discomfort. SPTs are associated with a favorable prognosis: After surgical resection, $95 \%$ of patients are disease-free, and mortality is $<2 \%$. However, SPTs also have a low potential for malignancy, and carry a $6-15 \%$ rate of recurrence or metastasis (5), with the liver being the most common metastatic site. There have been scattered reports of SPTs arising in extrapancreatic sites, including the retroperitoneum $(1,6,7)$, omentum $(8,9)$, ovary $(10-12)$ and gastroduodenal area (13). The most common extrapancreatic site is the mesocolon or omentum (1). In the present study, two unusual extrapancreatic SPTs. One case is an uncommon ovarian metastasis of an SPT, and the other is a retroperitoneal SPT. Both cases had a good outcome, as is the case with the majority of pancreatic SPTs.

\section{Case reports}

\section{Clinical features}

Case 1. A 22-year-old woman presented at the Emergency Department of our hospital with sudden abdominal pain; two years previously, the patient had been diagnosed with a pancreatic SPT that had extensively metastasized into the abdominal cavity. A review of her medical history revealed that the patient 
underwent interventional treatment, including a distal pancreatectomy, splenectomy, resection of liver segments IV and V, omental metastasectomy, and other resections of metastases. On this occasion, computed tomography (CT), performed on an Aquilon 64-layer Spiral CT scanner (Toshiba Medical Systems Corp., Tokyo, Japan), revealed a cystic and low-signal tumor located in the left ovary, measuring $12 \times 9 \mathrm{~cm}$. Levels of various tumor markers, including carbohydrate antigen (CA) 19-9, carcinoembryonic antigen and CA12.5, were normal. The patient underwent a left adnexectomy and a bilateral distal fimbriectomy, and did not experience a recurrence of the tumor. The patients remained alive over a follow-up period of 12 months.

Case 2. A 47-year-old woman was referred to our hospital due to slight pain in her upper left abdomen; the patient had lost $5 \mathrm{~kg}$ of weight since the onset of her symptoms. CT revealed a 16x14 cm heterogeneous, lobulated mass arising in the retroperitoneal area that was compressing the adjacent organs, including the bowel, left kidney and spleen. The pancreas appeared normal. Blood biochemistry, routine blood counts, and associated tumor markers were all within normal levels. The patient was scheduled for abdominal surgery, and the tumor was completely excised from the retroperitoneum. The patient has been without tumor recurrence for 14 months.

This study was approved by the Ethics Committees of the First Affiliated Hospital of Bengbu Medical College, and was conducted in accordance with the ethical guidelines of the Declaration of Helsinki.

Immunohistochemical staining. The collected specimens were fixed with $10 \%$ neutral buffered formalin, and embedded in paraffin blocks. Tissue blocks were cut into $4 \mu \mathrm{m}$ slides, deparaffinized in xylene, rehydrated with a graded alcohol series, and immunostained with the following mouse anti-human antibodies (all at a dilution of 1:100), against: Vimentin, epithelial membrane antigen (EMA), cytokeratin (CK), CK7, CD10, CD56, CD99, $\alpha 1$-antichymotrypsin (AACT), neuron-specific enolase (NSE), synaptophysin (Syn), chromogranin A (CgA), S-100, galectin-3 (GAL-3), calretinin, $\alpha$-inhibin, $\beta$-catenin, E-cadherin, cyclin D1, progesterone receptor (PR), estrogen receptor (ER) and Ki-67. Sections were stained using a streptavidin-peroxidase system (KIT-9720; Ultrasensitive TM S-P, Maixin Biotech, Inc., Fuzhou, China). The chromogen used was diaminobenzidine tetrahydrochloride substrate (employing a DAB kit; Maixin Biotech, Inc.), and sections were slightly counterstained with hematoxylin, dehydrated and mounted. Histochemical staining for Alcian Blue at $\mathrm{pH} 2.5$ was performed on sections from a block representing the predominant histology of each case. Immunohistochemical data are summarized in Table I.

\section{Gross and histological features}

Case 1. Macroscopically, the excised abdominal lesion measured 5x4x1 cm and was well-circumscribed, encapsulated, and exhibited a loss of content of its cystic mass.

Microscopically, the tumor had a fibrous capsule, and exhibited a combination of solid and cystic components, with cellular degenerative changes as well as pseudopapillary formation (Fig. 1A). Necrobiotic nests were readily visible. Tumor cells were monomorphic with ill-defined cytoplasmic limits,
Table I. Sources of the antibodies used in the immunohistochemistry analysis.

\begin{tabular}{ll}
\hline Source & \multicolumn{1}{c}{ Antibody } \\
\hline Vimentin & Monoclonal, clone V9 \\
EMA & Monoclonal, clone E29 \\
CK & Monoclonal, clone AE1/AE3 \\
CK7 & Monoclonal, clone OV-TL12/30 \\
CD10 & Monoclonal, clone 56C6 \\
CD56 & Monoclonal, clone 56C04 \\
CD99 & Monoclonal, clone 013 \\
AACT & Polyclonal \\
NSE & Monoclonal, clone E27 \\
Syn & Monoclonal, clone SYP02 \\
CgA & Monoclonal, clone 5p12 \\
S-100 & Monoclonal, clone 013 \\
GAL-3 & Monoclonal, clone 9C4 \\
Calretinin & Monoclonal, clone SP13 \\
$\alpha-i n h i b i n$ & Monoclonal, clone R1 \\
$\beta$-catenin & Monoclonal, clone CAT-5H10 \\
E-cadherin & Monoclonal, clone 4A2C7 \\
Cyclin D1 & Monoclonal, clone DCS-6 \\
PR & Monoclonal, clone 1A6 \\
ER & Monoclonal, clone 6F11 \\
Ki-67 & Monoclonal, clone MIB-1 \\
&
\end{tabular}

${ }^{a}$ All antibodies were obtained from Maixin Biotech, Inc. (Fuzhou, China), and were ready to use. EMA, epithelial membrane antigen; CK, cytokeratin; AACT, $\alpha 1$-antichymotrypsin; NSE, neuron-specific enolase; Syn, synaptophysin, CgA, chromogranin A; GAL-3, galectin-3; PR, progesterone receptor; ER, estrogen receptor.

with pale or eosinophilic cytoplasm. The nuclei were irregular, with nuclear membrane shrinkage, and small eccentric nucleoli were readily observed. The tumor exhibited an increased nuclear-to-cytoplasm (N/C) ratio and focal necrosis compared with primary SPTs of the pancreas. Focally, large numbers of intra- and extracellular hyaline globules were present (Fig. 1B). Pseudopapillary structures with a fibrovascular core were observed (Fig. 1C). Foamy histiocytes, cholesterol crystals and foreign body giant cell reactions were absent from this specimen.

Case 2. Macroscopically, the lesion was a lobulated large tumor accompanied by necrosis within a complete fibrous capsule. The cut surface was grey-red to yellow and multinodular, with slight hemorrhaging.

Microscopically, no clear pseudopapillae were observed. The solid area constituted a major portion, was rich in microvasculature, and revealed sheets of cells arranged around fibrovascular septa, forming a grid-like appearance. Few tumor cells with intracytoplasmic vacuoles were readily observed, and foamy tissue surrounding the solid area was evident (Fig. 2A). Certain areas exhibited monotonous cells with abundant eosinophilic cytoplasm, surrounded by delicate vessels resembling ependymal rosettes (Fig. 2B). Tumor cells revealed empty, 

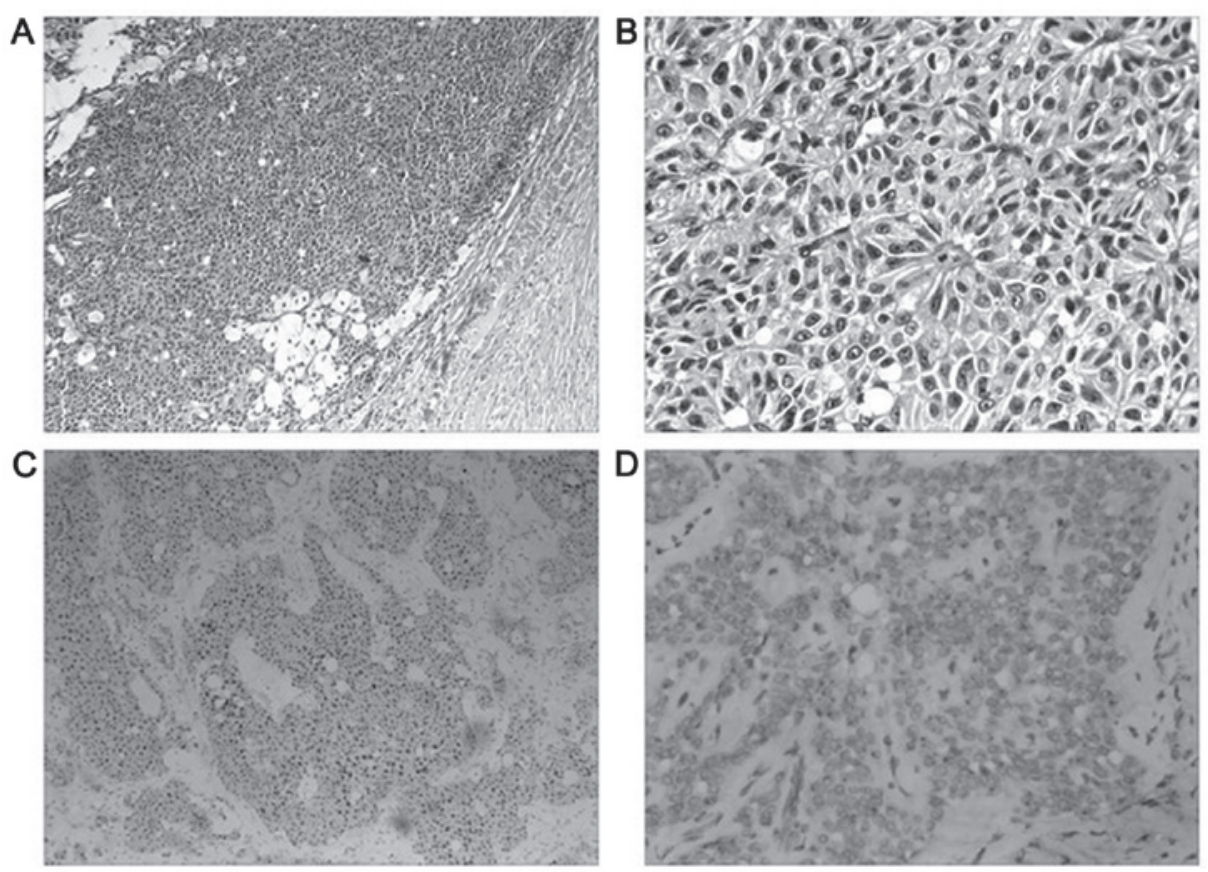

Figure 1. Histological and immunohistochemical features of the tumor from the patient described in case 1. (A) At low magnification, the tumor tissue presented a solid and pseudopapillary architecture (magnification, x100). (B) At higher magnification, large numbers of intra- and extracellular hyaline globules were observed (magnification, x400). (C) Pseudopapillary structures with a central fibrovascular core were observed (magnification, x100). (D) The nucleus was positive for progesterone receptor (magnification, $\mathrm{x} 400$ ).
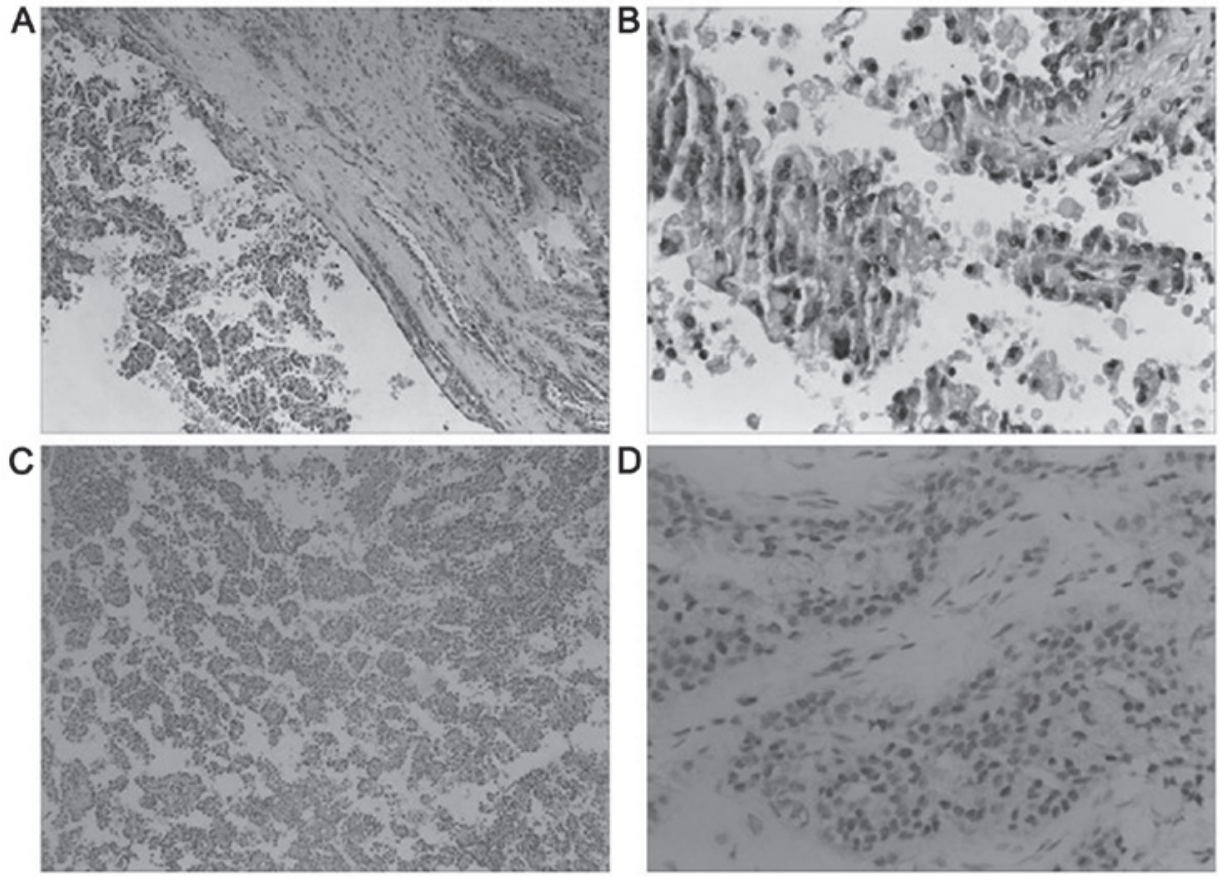

Figure 2. Histological and immunohistochemical features of the tumor from the patient described in case 2. (A) The solid areas revealed sheets and cords of cells arranged around fibrovascular septa, forming a grid-like appearance. A few tumor cells with intracytoplasmic vacuoles were observed, as well as a sheet of foamy tissue surrounding the solid area (magnification, x100). (B) Monotonous cells with abundant eosinophilic cytoplasm, surrounded by delicate vessels resembling ependymal rosettes are present (magnification, $x 400$ ). Both nuclear and cytoplasmic reactivity with (C) $\beta$-catenin (magnification, $x 100)$ and (D) CD99 (magnification, x400) exhibit a unique punctate staining pattern.

centrally localized nuclei, and vacuolated or faintly eosinophilic cytoplasm. Cellular atypia and mitotic figures were absent. In this case, an ectopic pancreatic component in the lesion was not identified.

\section{Immunohistochemical features}

Case 1. The immunohistochemical phenotype of the primary tumor was comparable with that of the ovarian metastasis. Immunohistochemical staining revealed that the tumor cells 
Table II. Immunohistochemistry profiles of cases 1 and 2.

\begin{tabular}{|c|c|c|}
\hline \multirow[b]{2}{*}{ Antibody source } & \multicolumn{2}{|c|}{ Case } \\
\hline & 1 & 2 \\
\hline Vimentin & + & + \\
\hline $\mathrm{CK}$ & + & - \\
\hline CK7 & + & - \\
\hline CD10 & + & + \\
\hline CD56 & + & + \\
\hline CD99 & + & + \\
\hline AACT & + & + \\
\hline NSE & + & - \\
\hline $\mathrm{CgA}$ & + & - \\
\hline GAL-3 & + & + \\
\hline$\beta$-catenin & + & + \\
\hline Cyclin D1 & + & + \\
\hline PR & + & - \\
\hline $\mathrm{Ki}-67$ & $<5 \%$ & $<2 \%$ \\
\hline EMA & - & - \\
\hline Syn & - & + \\
\hline E-cadherin & - & - \\
\hline Calretinin & - & - \\
\hline$\alpha$-inhibin & - & - \\
\hline ER & - & - \\
\hline S-100 & - & - \\
\hline
\end{tabular}

AACT, $\alpha 1$-antichymotrypsin; NSE, neuron-specific enolase; CgA, chromogranin A; GAL-3, galectin3; PR, progesterone receptor; EMA, epithelial membrane antigen; Syn, synaptophysin; ER, estrogen receptor.

were positive for vimentin, CK, CK7, CD10, CD56, CD99 (perinuclear punctate staining pattern), AACT, NSE, CgA, GAL-3, $\beta$-catenin (cytoplasmic and nuclear pattern), cyclin D1, and PR (Fig. 1D). The Ki-67 index was $<5 \%$. Other markers, including EMA, Syn, E-cadherin, calretinin, $\alpha$-inhibin, ER, and S-100 protein, were negative (Table II). The histological findings were compatible with the diagnosis of an ovarian metastasis of an SPT.

Case 2. Immunohistochemical findings revealed that the tumor cells were SPT cells, as they were characteristically positive for $\beta$-catenin (cytoplasmic and nuclear patterns; Fig. 2C), vimentin, CD10, CD56, CD99 (perinuclear punctate staining pattern; Fig. 2D), AACT, Syn, GAL-3 and cyclin D1. The Ki-67 index was $<2 \%$. Other markers, including CK, CK7, EMA, NSE, E-cadherin, $\alpha$-inhibin, CgA, calretinin, PR, ER and S-100, were negative (Table II). The final diagnosis was a primary retroperitoneal SPT.

\section{Discussion}

Solid pseudopapillary tumors of low malignant potential usually occur in the pancreas, and predominantly affect young females in their second or third decade of life (14). They occasionally occur in children $(2,14)$ and older females (13) or males (6). A review of 718 well-documented cases of pancreatic SPTs by Papavramidis et al (15) reported a male-to-female ratio of 1:9.78, with a mean age of 21.97 years (range, 2-85 years). The most common location was the tail of the pancreas. Patients generally presented with a palpable abdominal mass, and few patients presented with a ruptured pancreatic SPT following blunt abdominal trauma (14). Recurrence or metastasis is present in $6-15 \%$ of SPT cases $(5,15)$. The liver is the most common site of metastasis, followed by the lymph nodes and peritoneum (16). Lung metastasis was also reported in one previous study (17). In the present study, a rare metastatic ovarian tumor arising from an SPT was presented. A review of the English literature published between 1990 and 2013 revealed only 13 cases of extrapancreatic SPTs (1). There are only three reported cases of primary extrapancreatic SPTs arising in the retroperitoneal area $(1,6,7)$. Only one extrapancreatic SPT arising in the retroperitoneum without ectopic pancreatic tissue has been reported previously, by Miyazaki et al (7). A review of all the reports on extrapancreatic SPTs reveals that the tumors are normally benign, and are likely to have a favorable clinical course, similar to their pancreatic counterparts.

The mean SPT size is $6.08 \mathrm{~cm}$, and ranges from $0.5-34.5 \mathrm{~cm}$ (15). Microscopically, the tumor is characteristically a mixture of solid-cystic and pseudopapillary structures, and tumor cells are totally monomorphic with ill-defined cytoplasmic limits. The nuclei are uniform, and seldom exhibit nucleoli, whereas chromatin is finely dispersed. The chromatin may occasionally contain a single clear cytoplasmic vacuole (10), whereas nuclear pleomorphisms are rare. Intraand extracellular eosinophilic globules, foamy histiocytes, cholesterol crystals and foreign body giant cell reactions are commonly present in SPTs $(6,12,17)$. One SPT with prominent, atypical multinucleated giant tumor cells has been reported (18), and Tang et al (19) reported two cases of clinically aggressive SPTs of the pancreas with sarcomatoid carcinoma components. The authors indicated that unusual pathological features, including solid and diffuse growth patterns with extensive tumor necrosis and a high mitotic rate, may predict aggressiveness and clinical outcomes. Only three extrapancreatic SPTs that were associated with malignant behavior and resulted in metastasis have previously been reported $(9,11,13)$. They exhibited an increased N/C ratio, hyperchromasia, greater nuclear atypia and pleomorphism. Mitotic figures were detected more frequently compared with the primary tumor. One primary SPT of the ovary was even fatal (11). However, the mitotic indexes of metastatic and non-metastatic SPTs are not markedly different (19). Case 1 in the current study presented with evident necrotic nests, but without mitotic figures. Therefore, we hypothesized that cellular pleomorphism, as well as a slightly elevated mitotic rate, do not appear to have a marked impact on the behavior of SPTs. In case 2, the tumor lacked a pseudopapillary structure, which is otherwise common in SPTs of the pancreas. However, the solid area featured monotonous cells with abundant eosinophilic cytoplasm, surrounded by vessels resembling ependymal rosettes; this is consistent with the SPTs previously reported $(20,21)$.

According to the literature, the presence of positive N/C $\beta$-catenin in conjunction with the membrane loss of E-cadherin and perinuclear punctate staining pattern of CD99 are major 
diagnostic indicators (20,21). CD99 as an immunohistological marker for SPT was first studied by Li et al (22) in 2011. All of the SPTs in that study presented unique perinuclear punctate CD99 staining. Nuclear accumulation of $\beta$-catenin protein was present in $95 \%$ of cases, and activating $\beta$-catenin oncogenic mutations were identified in $90 \%$ of SPTs (21). This was also true of $\beta$-catenin in the two presented cases in the current study. Previously, Wnt signaling, mostly associated with $\beta$-catenin mutations, has been suggested to occupy a major role in the tumorigenesis of SPTs $(12,17,21)$. Immunoreactivity for nuclear PR was robust in $>80 \%$ of the cells $(23,24)$. Furthermore, a possible role for sex hormones in the histogenesis of SPTs has been suggested, although this is controversial, since $12.2 \%$ of SPTs occur in men (5) and PR is not always detected $(6,11)$. One of our own cases was negative for PR staining. Another report by Geers et al (23) revealed that SPT cells exhibit positive immunoreactivity for GAL-3, similarly to pancreatic duct cells. This led to the proposal of an hypothesis that SPTs have a duct cell origin (24). GAL-3 was detected in both case studies in the present study. The marker pattern, comprising the frequent expression of vimentin, NSE and $\alpha 1$-antitrypsin, together with negativity for CK, leads to the question of whether SPTs are mesenchymal, rather than epithelial tumors (23). In view of the striking female predominance of SPTs and the known approximation of the genital ridges to the pancreatic anlage during embryogenesis, it is possible that SPTs, including certain extrapancreatic SPTs identified in the retroperitoneal space, may arise from genital ridge/ovarian anlage-associated cells $(10,23)$.

Regarding the morphological overlap between SPTs and other neoplasms, the major differential diagnosis in case 1 of the present study is a yolk sac tumor (YST) of the ovary. YST, also known as an endodermal sinus tumor, is a rare malignant germ cell tumor that is able to emulate an SPT with multiple patterns of solid, as well as cystic, components, in addition to intra- and extracellular hyaline globules. However, Schiller-Duval bodies have only been identified in YSTs (25), and a key diagnostic feature of a YST is an elevated level of $\alpha$-fetoprotein, which distinguishes it from a metastatic ovarian tumor of an SPT. Mete et al (16) reported the first pancreatic SPT with ovarian metastasis, which was initially misdiagnosed as a bilateral sex-cord stromal tumor. Therefore, sex-cord stromal tumors should also be considered; however, it was possible to exclude this in the present study, since such tumors are positive for $\alpha$-inhibin and calretinin (which were negative in our cases) and lack pseudopapillary patterns (which were observed in one of our cases). In case 2 of the present study, the nested pattern of fibrovascular septa with a grid-like appearance may indicate paraganglioma; however, paragangliomas are positive for neuroendocrine markers, such as Syn and CgA, as well as for S-100. In addition, they have no pseudopapillary patterns and do not exhibit vimentin or AACT staining, and may therefore be ruled out.

In conclusion, SPTs with ovarian metastasis or primary retroperitoneal SPTs are rarely reported. The two cases in the current study presented with the identical pathological features and immunohistological phenotypes as pancreatic SPTs, with the exception of necrosis and an increased N/C ratio, which are more readily detected in metastatic ovarian neoplasms. The two cases exhibited nuclear and cytoplasmic reactivity with $\beta$-catenin, and complete loss of membrane E-cadherin staining. Furthermore, CD99 exhibited a unique perinuclear punctate staining pattern that was easily distinguishable from morphologically overlapping tumors, including YSTs of the ovary, sex-cord stromal tumors and paragangliomas. The good prognosis of the two unusual SPTs indicated that extrapancreatic SPTs are likely to have a favorable clinical course similar to their pancreatic counterparts; however, considering the $6.5 \%$ recurrence rate following resection in patients (14), a five-year follow-up period is recommended (5).

\section{Acknowledgements}

We would like to thank Editage Editing Services for their English language editing. We also thank Dr Di-chen Li and Professor Qun Xie (Department of Pathology, the First Affiliated Hospital of Bengbu Medical College) for their assistance with the histopathological and immunohistochemical stain evaluations. The present study was supported by the Natural Science Key Foundation of the Education Department in Anhui province, no. KJ2014A160.

\section{References}

1. Zhu H, Xia D, Wang B and Meng H: Extrapancreatic solid pseudopapillary neoplasm: Report of a case of primary retroperitoneal origin and review of the literature. Oncol Lett 5: 1501-1504, 2013.

2. Frantz VK: Tumor of the pancreas. In: Atlas of Tumor Pathology. Bumberg CW (ed). 1st edition. Armed Forces Institute of Pathology, Washington, pp32-33, 1959.

3. Klöppel G, Solcia E, Longnecker DS, Capella C and Sobin LH: World Health Organization International Histological Classification of Tumours: Histological Typing of Tumors of the Exocrine Pancreas. 2nd edition. Springer, Berlin, pp15-21, 1996.

4. Klöppel G, Hruban RH, Klimstra DS, et al: Solid-pseudopapillary tumor of pancreas. In: Bosman FT, Carneiro F, Hruban RH, Theise ND, eds. World Health Organization classification of tumours of the digestive system. IARC, Lyon, 327-330, 2010.

5. Law JK, Ahmed A, Singh VK, Akshintala VS, Olson MT, Raman SP, Ali SZ, Fishman EK, Kamel I, Canto MI, et al: A systematic review of solid-pseudopapillary neoplasms: Are these rare lesions? Pancreas 43: 331-337, 2014.

6. Klöppel G, Maurer R, Hofmann E, Lüthold K, Oscarson J, Forsby N, Ihse I, Ljungberg O and Heitz PU: Solid-cystic (papillary-cystic) tumours within and outside the pancreas in men: Report of two patients. Virchows Arch A Pathol Anat Histopathol 418: 179-183, 1991

7. Miyazaki Y, Miyajima A, Maeda T, Yuge K, Hasegawa M, Kosaka T, Kikuchi E, Kameyama K, Jinzaki M, Nakagawa K and Oya M: Extrapancreatic solid pseudopapillary tumor: Case report and review of the literature. Int J Clin Oncol 17: 165-168, 2012.

8. Fukunaga M: Pseudopapillary solid cystic tumor arising from an extrapancreatic site. Arch Pathol Lab Med 125: 1368-1371, 2001.

9. Hibi T, Ojima H, Sakamoto Y, Kosuge T, Shimada K, Sano T, Sakamoto M, Kitajima $M$ and Yamasaki S: A solid pseudopapillary tumor arising from the greater omentum followed by multiple metastases with increasing malignant potential. J Gastroenterol 41: 276-281, 2006.

10. Deshpande V, Oliva E and Young RH: Solid pseudopapillary neoplasm of the ovary: A report of 3 primary ovarian tumors resembling those of the pancreas. Am J Surg Pathol 34: 1514-1520, 2010.

11. Syriac S, Kesterson J, Izevbaye I, de Mesy Bentley KL, Lele S and Mhawech-Fauceglia P: Clinically aggressive primary solid pseudopapillary tumor of the ovary in a 45 -year-old woman. Ann Diagn Pathol 16: 498-503, 2012.

12. Kominami A, Fujino M, Murakami $H$ and Ito $M$ : $\beta$-catenin mutation in ovarian solid pseudopapillary neoplasm. Pathol Int 64: 460-464, 2014.

13. Walter T, Hommell-Fontaine J, Hervieu V, Adham M, Poncet G, Dumortier J, Lombard-Bohas C and Scoazec JY: Primary malignant solid pseudopapillary tumors of the gastroduodenal area. Clin Res Hepatol Gastroenterol 35: 227-233, 2011. 
14. Tajima Y, Kohara N, Maeda J, Inoue K, Kitasato A, Natsuda K, Irie J, Adachi T, Kuroki T, Eguchi S and Kanematsu T: Peritoneal and nodal recurrence 7 years after the excision of a ruptured solid pseudopapillary neoplasm of the pancreas: Report of a case. Surg Today 42: 776-780, 2012

15. Papavramidis T and Papavramidis S: Solid pseudopapillary tumors of the pancreas: Review of 718 patients reported in English literature. J Am Coll Surg 200: 965-972, 2005.

16. Mete Ö, Yegen G, Güllüoglu MG, Kapran Y and Klöppel G: An unusual clinical presentation of pancreatic solid pseudopapillary tumor with ovarian metastases: A diagnostic dilemma. Int J Surg Pathol 19: 342-345, 2011.

17. Takahashi Y, Fukusato T, Aita K, Toida S, Fukushima J, Imamura T, Tanaka F, Amano H, Takada T and Mori S: Solid pseudopapillary tumor of the pancreas with metastases to the lung and liver. Pathol Int 55: 792-796, 2005.

18. Li L, Othman M, Rashid A, Wang H, Li Z, Katz MH, Lee JE, Pisters PW, Abbruzzese JL, Fleming JB and Wang H: Solid pseudopapillary neoplasm of the pancreas with prominent atypical multinucleated giant tumour cells. Histopathology 62: 465-467, 2013

19. Tang LH, Aydin H, Brennan MF and Klimstra DS: Clinically aggressive solid pseudopapillary tumors of the pancreas: A report of two cases with components of undifferentiated carcinoma and a comparative clinicopathologic analysis of 34 conventional cases. Am J Surg Pathol 29: 512-519, 2005.
20. Bhatnagar R, Olson MT, Fishman EK, Hruban RH, Lennon AM and Ali SZ: Solid-pseudopapillary neoplasm of the pancreas: Cytomorphologic findings and literature review. Acta Cytol 58: 347-355, 2014.

21. Abraham SC, Klimstra DS, Wilentz RE, Yeo CJ, Conlon K, Brennan M, Cameron JL, Wu TT and Hruban RH: Solid-pseudopapillary tumors of the pancreas are genetically distinct from pancreatic ductal adenocarcinomas and almost always harbor beta-catenin mutations. Am J Pathol 160: 1361-1369, 2002.

22. Li L, Li J, Hao C, Zhang C, Mu K, Wang Y and Zhang T: Immunohistochemical evaluation of solid pseudopapillary tumors of the pancreas: The expression pattern of CD99 is highly unique. Cancer Lett 310: 9-144, 2011.

23. Geers C, Moulin P, Gigot JF, Weynand B, Deprez P, Rahier J and Sempoux C: Solid and pseudopapillary tumor of the pancreas-review and new insights into pathogenesis. Am J Surg Pathol 30: 1243-1239, 2006

24. Kosmahl M, Seada LS, Jänig U, Harms D and Klöppel G: Solid-pseudopapillary tumor of the pancreas: Its origin revisited. Virchows Arch 436: 473-480, 2000.

25. Van Thielen T, Degryse H and Coeman D: Yolk sac tumor of the ovary. JBR-BTR 96: 256-7, 2013. 